\title{
Le devenir prochain de la pluie: infiltration ou ruissellement
}

\author{
Rainwater infiltration and runoff
}

\author{
Claude Thirriot \\ Professeur à l'Institut National Polytechnique de Toulouse \\ Professeur à l'École Nationale d'Ingénieurs de Tunis
}

\section{Introduction}

Il pleut. Averse impulsive et impétueuse méditerranéenne ou crachin breton doux et obstiné, que devient cette eau? Le bilan hydrologique est d'une limpidité de source :

$$
P=E+I+R
$$

L'eau précipitée $P$ s'évapore, s'infliltre et ruisselle. Mais comment se fait le partage entre ces trois destinations?

Cette question concrète simple et familière depuis l'enfance est en fait redoutable car elle a la complexité de la nature.

Nous essaierons tout d'abord de distinguer les différentes échelles de temps et d'espace intervenant explicitement ou implicitement dans l'observation puis l'étude du phénomène.

Puis, sur des exemples simples, nous tenterons de cerner l'influence des différents paramètres.

Ensuite, moyennant des hypothèses simples, nous examinerons quelques modèles.

\section{Les échelles d'observation}

Les variables sont le temps et les coordonnées d'espace qui peut être à trois dimensions.

L'échelle de temps prise en compte dans le bilan hydrologique peut-être la durée de la pluie (de quelques minutes à quelques heures), l'intervalle entre deux épisodes pluvieux nettement séparés (quelques jours) ou l'année hydrologique.

Suivant le choix de l'échelle de référence de temps, le bilan sera interprêté différemment.

Sur la durée de la pluie, il n'y aura pas d'évaporation effective appréciable mais par contre il $\mathrm{y}$ aura inter- ception par les arbres, la végétation superficielle ou un tapis de feuilles mortes d'une quantité d'eau qui sera ensuite reprise rapidement par évaporation.

Cette évaporation peut être immédiate pour les premières gouttes d'un orage avorté qui tombent sur un sol surchauffé.

Sur un terrain peu perméable, par forte pluie, des flaques peuvent se former très vite qui seront bues ensuite par le soleil regaillardi.

Cette évaporation différée est à prendre en compte dans le bilan sur la durée même de la pluie avant d'essayer de répartir l'eau tombée en infiltration et ruissellement.

A l'échelle de temps de quelques jours, il est aussi nécessaire de tenir compte de l'évaporation différée de l'eau retenue à la surface du sol ou infiltrée sur une très faible épaisseur et que nous classerons dans la rétention superficielle. C'est ce qui arrive, par exemple, sur les mottes d'un labour marneux qui sont imbibées d'eau à la périphérie sur quelques millimètres.

Lorsque l'échelle de temps s'allonge, l'extension de la zone de rétention superficielle s'accroît aussi. A la limite, on peut la cerner par la profondeur de la couche de sol soumise à l'évapotranspiration (ou à l'évaporation sur sol nu). Dans le bilan hydrologique, l'infiltration concerne alors la partie de la pluie qui va atteindre la nappe phréatique.

Même distinction peut être faite pour le ruissellement en conjugeant cette fois échelle de temps et échelle d'espace.

Suivant les accidents du terrain, l'eau de pluie peut commencer à ruisseler puis à s'infiltrer à partir des petites rigoles naturelles tout comme en irrigation. L'exemple exacerbé est celui des oueds descendant de l'Atlas qui disparaissent dans les alluvions parfois au bout de quelques centaines de kilomètres de course en crue. 
A l'opposé, ne doit-on pas classer, dans le ruissellement à grande échelle de distance, la résurgence rapide dans les fossés ou les ruisseaux, de l'eau infiltrée? A la limite, pour un vaste système hydrogéologique ouvert sur l'océan, dans le bilan même annuel, l'infiltration ne représentera que la variation de stock sensible aux aléas météorologiques pluriannuels.

Ainsi donc, face au dilemne infiltration ou ruissellement, force est de constater l'ubiquité ou tout au moins l'ambiguité de la réponse suivant les échelles d'observation de temps et d'espace : ce qui sera infiltration pour le jardinier du dimanche amoureux de son semis de radis n'aura rien à voir avec les mouvements de stocks d'eau souterrains manipulés par l'hydrogéologue préoccupé de bilan à l'échelle décennale.

Dans ce qui suit, nous suivrons un itinéraire allant $\mathrm{du}$ lysimètre au continent en insistant, dans la mesure du possible, sur les effets d'échelle.

\section{Le phénomène à l'échelle de l'échantillon}

\section{Le seuil de ruissellement}

Les paramètres de l'alternative infiltration-ruissellement sont tout de même assez nombreux : caractéristiques du milieu poreux (pente de la surface et sa rugosité éventuellement due à la couverture végétale, perméabilité ou plus finement architecture de l'espace poreux) et paramètres de la pluie (intensité, durée, intervalle entre précipitations successives).

Le ruissellement ne peut commencer que lorsque le sol ne peut plus absorber tout le débit de la pluie (il s'agit évidemment de la pluie nette) et ceci indépendamment de la pente.

\section{Les hypothèses d'une première approche}

Puisque la pente de la surface n'intervient pas directement dans l'infiltration, nous supposerons horizontale la limite superieure du milieu poreux réputé d'abord homogène.

Le phénomène d'infiltration de la pluie est un phénomène d'écoulement diphasique. En effet, l'eau va rentrer dans des pores où se trouve de l'air. Mais, bien souvent, dans une première approche (que l'on critiquera par la suite) on suppose négligeable l'influence de l'air et l'on utilisera la manière habituelle des milieux non saturés.

Avec ces hypothèses d'homogénéité et de surface du sol horizontale, l'écoulement peut être considéré comme vertical donc le problème est à une seule dimension d'espace.

\section{Brefs rappels sur les milieux non saturés}

L'eau n'occupe pas tous les pores mais elle constitue un milieu continu dans lequel la pression varie sans discontinuité. Par contre, à la traversée de l'interface séparant l'eau et l'air contigü, il existe une discontinuité de pression appelée pression capillaire $p_{c}$ et dont on peut avoir une idée à partir de la loi de Jurin

$$
p_{c}=p_{1}-p_{0}=-\frac{4 A \cos \theta}{d_{e}}
$$

avec :

$p_{0}$ : pression dans l'air supposée égale à la pression atmosphérique (hypothèse qui sera discutée plus tard)

$p_{1}$ : pression dans l'eau ou pression interstitielle

$A$ : tension superficielle du système air/eau (elle vaut environ $0,075 \mathrm{~N} / \mathrm{m}$ aux conditions habituelles de température).

$d_{e}$ : diamètre du capillaire équivalent au col de pores de frontière entre l'air et l'eau

$\theta$ : angle de raccordement du ménisque avec la paroi compté du côté de l'eau (comme l'eau est supposée liquide parfaitement mouillant $\theta=0$ ).

La pression dans l'eau est donc inférieure à la pression de l'air (c'est-à-dire à la pression atmosphérique). On parle de succion $\psi$ tel que $\psi=p_{c} / \rho g$.

Le diamètre $d_{e}$ à prendre en compte va dépendre de la quantité d'eau contenue dans le sol qui est repérée par la teneur en eau $\theta=\Omega_{\epsilon} / \Omega_{\text {tot }}$, rapport du volume d'eau sur le volume géométrique de la portion de milieu poreux considéré ou encore par le degré de saturation $S=\theta / P, P$ étant la porosité $(S \in[0,1])$.

Lorsqu'il y a peu d'eau, elle est réfugiée dans les petits canicules formés par la succession très tortueuse des petits pores (qui peuvent éventuellement en emprisonner un gros). La valeur absolue de la succion est alors très forte. Par contre, s'il y a abondance d'eau, ce sont les courbures des ménisques dans les plus gros pores qui fixent la valeur absolue de la succion qui est alors très faible.

On comprend donc que l'on obtienne des graphes $\psi(\theta)$ analogues à ceux présentés sur la figure 1; un phénomène d'hystérésis apparaît entre le remplissage (ou imbibition) et le drainage.

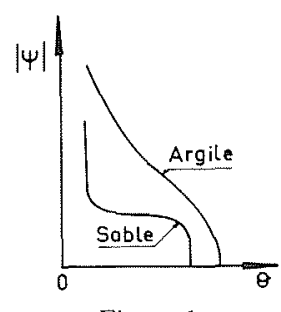

Figure 1

Pour ce qui concerne l'écoulement, on applique encore au milieu non saturé la loi de Darcy mais la perméabilité dépend de $\theta$ :

$$
\vec{q}=-K(\theta) \overrightarrow{\operatorname{grad}}(\psi+Z)
$$

\section{$Z$ : cote du point.}

Pour certain sables, G. Vachaud a obtenu, à Grenoble, la loi suivante :

$$
K(\theta)=K(P)\left(\frac{\theta-\theta_{\min }}{P-\theta_{\min }}\right)^{3,5}
$$

Dans le cas de la seule dimension verticale, on obtient finalement :

$$
\frac{\partial \theta}{\partial t}=\frac{\partial}{\partial Z}\left[K(\theta) \frac{\partial}{\partial Z}(\psi(\theta)+Z)\right]
$$


Etude théorique de l'infiltration en terrain homogène

\section{Les conditions aux limites}

A la surface du sol, la condition de liaison avec l'atmosphère est le hyétogramme.

Nous supposerons qu'il s'agit d'une fonction créneau, d'intensité $q_{0}$ et de durée $T_{0}$.

Tant que l'eau précipitée peut s'infiltrer, la condition à $Z=0$ est donc $q=q_{0}$.

Ensuite, une partie de l'eau de pluie s'accumule en surface et la condition à $Z=0$ est une condition de pression imposée par la hauteur de la lame d'eau accumulée. Nous admettrons, en première approximation raisonnable, que cette hauteur reste faible et, donc, qu'il règne à la surface du terrain la pression atmosphérique.

Les conditions initiales correspondent à $q=0$, c'està-dire à l'équilibre statique sous l'effet de la gravité :

$$
\psi+Z=\text { cte }
$$

$Z$ compté positif dans le sens vertical ascendant.

\section{Etude simplifiée dans le cas du sol immédiatement noyé}

On suppose l'averse si intense que la couche superficielle du sol ne puisse absorber tout le débit.

Si on suppose que $\theta\left(0, Z_{f}\right)$ est constant, $\psi\left(0, Z_{f}\right)$ est aussi constant et on retrouve la formulation de Green Ampt proposée dès 1911:

$$
\frac{K\left(\theta_{\max }\right) \cdot t}{\theta_{\max }-\theta_{0}}=-Z_{f}+\psi_{0} \log \left(1+\frac{Z_{f}}{\psi_{0}}\right)
$$

$Z_{f}$ altitude du front $\left(Z_{f}\right.$ est négatif).

Le débit instantané infiltré vérifie donc l'équation :

$$
\begin{array}{r}
\frac{K\left(\theta_{\max }\right) t}{\left|\psi_{0}\right|\left(\theta_{\max }-\theta_{0}\right)}=-\log \left(1-\frac{1}{1+\frac{q(0, t)}{K\left(\theta_{\max }\right)}}\right)- \\
-\frac{1}{1+\frac{q(0, t)}{K\left(\theta_{\max }\right)}}
\end{array}
$$

\section{Bref aperçu de formules empiriques du débit d'infiltration}

Dès 1921, Gardner et Witsoe proposaient une formule d'avancement du front d'humidité dont on peut déduire une formule de débit analogue à celle proposée par Horton en 1940 :

$$
q(0, t)=q(0, \infty)+[q(0,0)-q(0, \infty)] e^{-\beta t}
$$

On retrouve là, la sempiternelle évolution exponentielle, comme dans les lois de tarissement, et qui est dans le doute la meilleure appréciation approchée des comportements naturels à effet diffusif ou dissipatif.
En 1932, Kostiakov proposa une formule monome :

$$
q(0, t)=\alpha K t^{\alpha-1}
$$

qui conduit à l'expression du volume infiltré :

$$
\Omega=K t^{\alpha}
$$

La signification physique des paramètres n'est pas évidente. En comparant avec la formule de Green-Ampt, on voit qu'il faudrait accepter une variation de $\alpha$ dans le temps:

$$
\alpha \rightarrow \frac{1}{2} \text { si } t \rightarrow 0 \quad \text { et } \quad \alpha \rightarrow 1 \text { si } t \rightarrow \infty
$$

En gros, avec une seule valeur constante de $\alpha$ :

$\alpha$ tend vers $1 / 2$ pour des sols argileux à fort effet capillaire ;

$\alpha$ tend vers 1 pour les milieux sableux où l'effet gravitaire l'emporte sur l'effet capillaire.

\section{La théorie de Philip}

Depuis près de vingt cinq ans, Philip a fourni sur le sujet une production de rapports nourrie, souvent originale, toujours lucide.

Il obtient par exemple comme expression pour le débit instantané d'infiltration

$$
\begin{aligned}
-q(0, t)= & \frac{d \Omega}{d t}=\frac{1}{2 \sqrt{t}} \int_{\theta_{0}}^{\theta_{\max }} \Phi d \theta+\mathrm{K}\left(\theta_{0}\right)+ \\
& +\int_{\theta_{0}}^{\theta_{\max }} \chi d \theta+\frac{3}{2} t^{1 / 2} \zeta d \theta+\cdots
\end{aligned}
$$

avec $\phi, \chi, \zeta$ fonctions de $\theta$ seul.

De son résultat, Philip a tiré de fructueuses conclusions :

1) le débit d'infiltration évolue bien au démarrage comme $1 / \sqrt{t}$. Vu l'importance du coefficient de $1 / \sqrt{t}$, il lui a donné un nom: la sorptivité $S$

$$
S=\frac{1}{2} \int_{\theta_{0}}^{\theta_{\max }} \Phi d \theta
$$

$S^{2}$ a la dimension d'une diffusivité.

2) Des exemples numériques lui ont montré que la solution de Green-Ampt fondée sur le front abrupt d'humidité était une excellente approximation.

3) $\mathrm{Vu}$ la proximité prouvée des résultats des deux théories, on peut donc retenir que $S$ varie approximativement comme :

$$
\left[\left|\psi_{0}\right|\left(\theta_{\max }-\theta_{0}\right)\right]^{1 / 2}
$$

\section{Les modèles numériques}

Sans vouloir entrer dans le détail, donnons un bref aperçu des approches possibles. 
Soit l'équation :

$$
\frac{\partial \theta}{\partial t}=\frac{\partial}{\partial Z}\left(K(\theta) \frac{d \psi}{d \theta} \frac{\partial \theta}{\partial Z}\right)+\frac{\partial K}{\partial \theta} \frac{\partial \theta}{\partial Z}
$$

ou encore, en utilisant la transformation de Kirchoff :

$$
\begin{gathered}
\mathrm{F}=\int_{\theta}^{\theta} D(\theta) d \theta \quad \text { d'où : } \quad \theta=G(F) \\
\frac{\partial F}{\partial t}=D^{\prime}(F) \frac{\partial^{2} F}{\partial Z^{2}}+C(F) \frac{\partial F}{\partial Z}
\end{gathered}
$$

Il reste une forte non linéarité de l'opérateur mais surtout l'incidence du terme convectif.

Pour pallier cette dernière difficulté, plusieurs remèdes ont été essayés qui ont pratiquement en commun le principe suivant:

- travailler en variable de Lagrange pour résoudre le problème de la convection ou de l'onde simple et utiliser ensuite la variable d'Euler pour étudier le phénomène de diffusion.

\section{Etudes expérimentales}

Il est hors de propos de passer en revue tous les comptes rendus d'expériences réalisées sur colonne d'infiltration au laboratoire ou sur le terrain. Mais nous voulons rendre d'abord un hommage ému et chaleureux à Féodoroff qui, en France, fut un pionnier, travaillant lors de la préparation de sa thèse avec des moyens rudimentaires qui paraitraient aujourd'hui dérisoires.

Georges Vachaud, empêché par une mission en Côte d'Ivoire, aurait dû nous parler lui-même des travaux de son Laboratoire de l'Institut de Mécanique de Grenoble (I.M.G.). Grâce à lui, à son ingéniosité et son opiniâtreté, les laboratoires et les équipes françaises de terrain ont pu peu à peu utiliser une métrologie moderne pour l'étude des écoulements en milieux non saturés.

Sur l'incitation de R. Degallier et de P. Mutin, qui avaient installé une station d'observation sur le site de Mielhan, nous avons, à Toulouse, transporté le terrain naturel dans le laboratoire. Avec W. Rizk, A Djoucka, R. Pizarro et P. Poulain, nous avons examiné les conséquences de succession de pluies sur des massifs argileux de près de trois mètres de hauteur.

La figure 2 donne une idée des phénomènes observés.

\section{Influence de l'air dans l'infiltration verticale en milieu homogène}

Le schéma le plus plausible de la coexistence de l'eau et de l'air est le suivant : l'eau, fluide mouillant, choisit de se faufiler d'abord dans les chenaux fermés de pores les plus petits, l'air fluide non mouillant va circuler de gros pores en gros pores et il est parfois piégé par un environnement de petits pores investis par l'eau. Cette image des chemins préférentiels permet aussi de définir des perméabilités respectives pour l'eau et pour l'air qui dépendent de l'espace occupé par chacun des fluides.

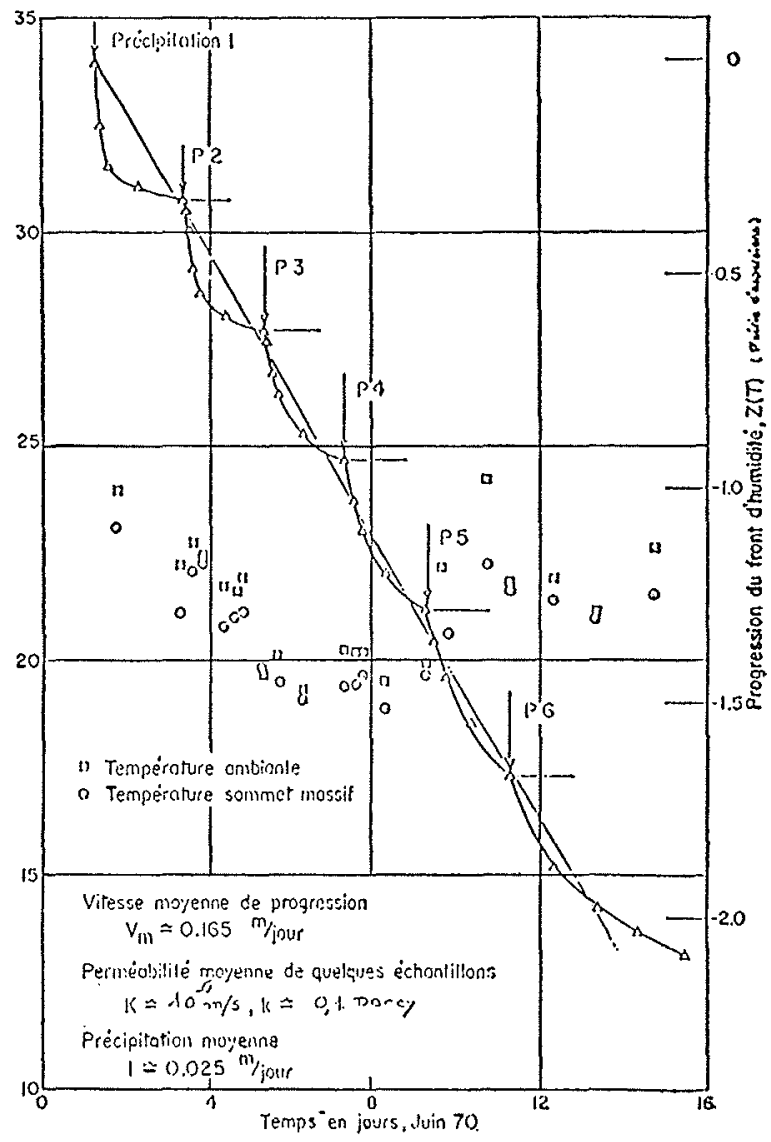

Figure 2

Soient $K_{0}\left(\theta_{0}\right)$ et $K_{1}\left(\theta_{1}\right)$ les perméabilités fonction des teneurs $\theta_{0}$ en air et $\theta_{1}$ en eau $\left(\theta_{0}+\theta_{1}=P\right)$.

Comme le font habituellement les pétroliers, on introduit les perméabilités relatives par rapport à la perméabilité géométrique $k$ :

$$
k_{i}^{\prime}\left(\theta_{i}\right)=\frac{K_{i}\left(\theta_{i}\right)}{K_{i}(P)}=\frac{K_{i}\left(\theta_{i}\right)}{g \frac{k}{\nu_{i}}}
$$

Les graphes $k_{i}^{\prime}\left(\theta_{i}\right)$ ont l'allure indiquée sur la figure 3 .

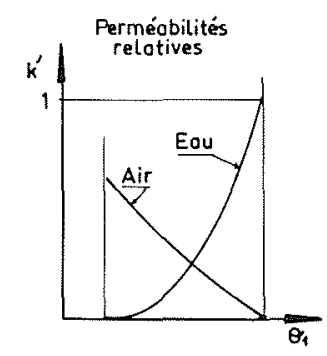

Figure 3

On admet que la loi de Darcy peut se généraliser sous la forme :

avec

$$
\overrightarrow{q_{i}}=-k_{i}^{\prime}\left(\theta_{i}\right) \frac{g k}{\eta_{i}} \overrightarrow{\operatorname{grad}}\left(p_{i}+\rho_{i} g Z\right)
$$

$$
p_{1}=p_{0}+\rho_{1} g\left(\theta_{1}\right)
$$


Reste à faire intervenir l'équation de continuité. Bien que cela paraisse à priori audacieux, on peut considérer avec une bonne approximation que l'air se comporte comme un fluide incompressible. Alors, les flux volumiques sont tels que :

$$
\begin{gathered}
\qquad \overrightarrow{q_{0}}+\overrightarrow{q_{1}}=0 \\
\text { D'où } \\
q_{1}=-\frac{k \rho_{1} g}{\frac{\eta_{0}}{k_{0}^{\prime}}+\frac{\eta_{1}}{k_{1}^{\prime}}} \frac{\partial}{\partial Z}\left[\psi\left(\theta_{1}\right)+\left(\frac{\rho_{1}-\rho_{0}}{\rho_{1}}\right) Z\right]
\end{gathered}
$$

Paradoxalement par rapport à la théorie "monophasique" du milieu non saturé, la perméabilité chute au voisinage de la saturation en eau. Ceci s'explique par la difficulté de l'air à circuler. Mais il faudrait alors tenir compte de la compressibilité de l'air.

Comme on le voit, la prise en compte de l'air permet de proposer une explication (approximative) du fait qu'un milieu noyé en surface ne soit pas saturé derrière le front d'humidité.

Il y a quelques années, le professeur H. MorelSeytoux de l'Université du Colorado a donné une excellente analyse détaillée de ces phénomènes.

\section{Infiltration à partir de réseaux superficiels}

\section{Les rigoles}

L'eau qui court s'infiltre à travers la paroi de la rigole représentant ainsi, à échelle réduite, ce qui se passe dans un oued en crue ou dans une rigole d'irrigation. Mais pour ce qui concerne l'infiltration, on peut considérer le problème de l'écoulement dans un plan vertical normal à la direction longitudinale de la rigole.

\section{Les fentes de retrait}

Craquelures superficielles ou failles profondes comme les racines provenarıl du dessèchement superficiel de sols truffés d'argile gonflante comme la montmarillonite, les fentes de retrait constituent un système privilégié de pénétration pour l'eau de pluie. On pourrait être tenté de les assimiler à un milieu karstique à double porosité : la porosité de fissure ou macroporosité et la porosité d'interstices ou microporosité. Mais il s'agirait d'un karst vivant qui fermerait ses machoires sur l'eau emprisonnée. Il faudrait alors considérer le sol comme un milieu biologique au comportement rhéologique complexe et capricieux.

A ces fentes de dessèchement, on peut aussi associer les trous longiformes laissés par la faune invertébrée et les racines décomposées.

Du point de vue de l'hydrodynamique, aux deux modes macroscopique et microscopique de porosité vont correspondre deux étapes dans le temps. Première étape : les fentes se gorgent d'eau - Deuxième étape : cette eau imbibe les zones voisines profitant de la mise en charge pour accélérer le mouvement si les fissures sont de profondeur appréciable par rapport à la hauteur d'eau équivalente capillaire moyenne : $\widetilde{\psi}$.
La formulation mathématique du système hybride discret pour les fentes, continu pour les pores, va être assez délicate mais on peut simplifier en supposant la juxtaposition de deux milieux à perméabilités différentes avec coefficient d'échange.

Soient $\theta_{1}$ la quantité d'eau contenue dans les fentes par unité de volume et $\theta_{2}$ la quantité contenue dans les pores, $Q_{12}$ le débit d'échange aussi par unité de volume.

En admettant que la loi de Darcy soit valable pour l'écoulement dans le milieu fissuré, il vient :

$$
\begin{aligned}
& \frac{\partial \theta_{1}}{\partial t}=\frac{\partial}{\partial Z}\left[K_{1}\left(\theta_{1}\right) \frac{\partial}{\partial Z}\left(\psi_{1}+Z\right)\right]-Q_{12} \\
& \frac{\partial \theta_{2}}{\partial t}=Q_{12}
\end{aligned}
$$

Pour l'évaluation de $Q_{12}$ nous admettrons que :

$$
Q_{12}=\alpha\left[\psi_{1}\left(\theta_{1}\right)-\psi_{2}\left(\theta_{2}\right)\right]
$$

Avec ce modèle mathématique, on retrouve-là dans le sens vertical un système d'équation analogue à celui qui régit l'évolution de la hauteur d'eau dans les rigoles infiltrantes.

\section{Le phénomène à l'échelle de la parcelle}

\section{Homogénéité et changement d'échelle}

On parle encore d'homogénéité à l'échelle de la parcelle : même couverture végétale, pente uniforme, granulométries analogues en différents points du domaine. Mais cette homogénéité globale est tissée des hétérogénéités à l'échelle de l'échantillon : petites aspérités du sol provenant déjà du travail d'érosion de l'eau ou des boursoufflures des racines, présence localisée des touffes d'herbe ou des plantes, rigoles signalant les chemins préférentiels d'écoulement. Tout cela est intégré à l'échelle de la parcelle comme les discontinuités des points et des lignes dans une portion d'image d'écran de télévision.

\section{Les paramètres de l'hydrodynamique du phénomène}

Reprenons la chronique d'un épisode pluvieux supposé à intensité constante :

1) Au début de la pluie, il y a rétention superficielle. Les premières gouttes mouillent les feuilles, les obstacles, l'épiderme du sol.

2) Lorsque l'eau atteint la surface du sol en abondance, l'infiltration commence et elle va se poursuivre seule jusqu'à l'instant où la capacité d'absorption devient inférieu re à l'intensité de la pluie.

3) Alors, apparaissent des flaques isolées si le terrain est plat.

4) Le ruissellement commence avec les premières rigoles, torrents liliputiens, ou avec le débordement des flaques.

5) Si la pluie dure assez, le phénomène peut se stabiliser. Le débit d'infiltration atteint sa valeur asymptotique. Le ruissellement est tout juste le complément de ce débit par rapport à l'intensité de la pluie. 
6) La pluie cesse - Le ruissellement essore la surface du sol à partir des zones amont - La surface horizontale d'infiltration diminue progressivement, donc le débit global infiltré aussi. Le débit ruisselé à l'exutoire diminue aussi car il n'est alors que le produit de la vidange du volume d'eau qui est à la surface du sol au moment où la pluie s'arrête, volume dont une partie s'infiltre d'ailleurs.

\section{Le régime permanent}

$\mathrm{Au}$ point de vue hydrodynamique, ce qui caractériserait le mieux le ruissellement c'est la hauteur d'eau moyenne de la lame ruisselée. En fait, à cause des hétérogénéités, on ne peut avoir qu'une valeur moyenne fictive. Cette valeur caractérisera le stock d'eau en place sur la parcelle en régime permanent et qui devra être évacué ensuite par ruissellement et infiltration à la fin de la pluie.

Donc, en définitive, ce stock d'eau dépend de la conductance hydraulique ou débitance au ruissellement,

Le débit à l'exutoire pourrait s'écrire :

$$
Q=\alpha \sqrt{\Sigma} \frac{J H}{n}
$$

avec :

$\Sigma$ surface de la parcelle

$J$ pente moyenne de la surface du sol

$H$ hauteur moyenne de la lame ruisselée

$n$ coefficient de rugosité inverse d'une perméabilité

$\alpha$ coefficient de forme supposé constant pour une parcelle

$n$ varie suivant la préparation du sol et la nature de la couverture végétale.

A débit fixe, $H$ varie approximativement comme $n / J$

\section{Le régime transitoire initial}

Il est caractérisé par deux temps :

- le temps de rétention $t_{r}$ correspondant aux étapes 1 et 2 de la chronique;

- le temps d'établissement du régime permanent $t_{e}$ du ruissellement dont une minorante est le temps de concentration tel qu'il est defini habituellement.

\section{Le régime transitoire de tarissement}

Il peut être caractérisé globalement par un temps de tarissement $t_{t}$.

Sans nourrir beaucoup d'illusions, on peut essayer de construire un modèle mathématique du régime transitoire.

Soit :

$\Sigma(t)$ la surface infiltrante à l'instant $t$

$Q(t)$ le débit de ruissellement à l'exutoire

$\Omega(t)=\Sigma(t) \cdot H(t)$ le volume encore accumulé à l'instant $t$.

$V=\beta \frac{J}{n}$ la vitesse des particules liquides.

Supposons que la ligne de démarcation entre partie noyée et partie dénoyée de la surface se déplace à la vitesse $V$. Comme $\sqrt{\Sigma}$ représente une distance à l'exutoire, on peut écrire :

$$
\frac{d \sqrt{\Sigma}}{d t}=-\gamma V=-\gamma^{\prime} \frac{J}{n}
$$

D'où :

$$
H=\frac{K\left(\theta_{\max }\right)}{\gamma V(1+a)} \sqrt{\Sigma} \quad \text { et } \quad t_{t}=\frac{n \sqrt{\Sigma_{0}}}{\gamma^{\prime} J}
$$

\section{Exemple d'étude expérimentale sur le terrain}

Pour illustrer le phénomène, nous utiliserons large. ment les campagnes réalisées par une équipe de l'Orstom sur des parcelles expérimentales en Haute-Volta et en Côte d'Ivoire pour juger des facteurs de ruissellement sous pluies simulées. Un article de synthèse a été présenté par A. Lafforgue et $\mathrm{E}$. Naah sur les expériences réalisées à Adiopodoumé sur des sols ferrallitiques sableux très homogènes aux caractéristiques pédolo. giques bien connues. Douze parcelles de $50 \mathrm{~m}^{2}$ (largeur $5 \mathrm{~m}$, longueur $10 \mathrm{~m}$ ) ont été observées, trois en sol nu et neuf en culture d'ananas.

\section{L'étude du phénomène à l'échelle régionale ou continentale}

Si l'on accroît la taille de la parcelle, on passe progressivement à l'échelle de bassin versant en abandonnant la circonstance d'homogénéité de nature de sol et de couverture végétale. Bien des essais ont été entrepris sur les bassins versants expérimentaux avec l'ambition de mieux cerner la relation pluie-débit en prenant en compte l'infiltration. Nous avons pu nous-même apprécier les efforts développés par une équipe du GERSAR et, tout particulièrement par S. Airault, dans l'étude du bassin de Dardaillon, près de Lunel. La question la plus délicate est de caractériser l'infiltration et l'évolution du stock d'eau souterrain simplement à partir d'une ou quelques observations ponctuelles verticales à l'aide de sondes neutroniques. La tentative est courageuse et intéressante mais pas encore suffisamment probante.

Alors que dire de l'exploit de Lin, Alfano et Bock de l'Université du Connecticut qui ont proposé, au demier congrès de l'AIRH, un modèle hydrologique à l'échelle du continent américain tout entier?

Ces auteurs ont découpé ce continent en éléments de 400 à $500 \mathrm{~km}$ de côté. Le sol est représenté par trois couches pour lesquelles sont suivis les bilans de masse et de chaleur en tenant compte des évènements météorologiques et de l'évapotranspiration. L'ensemble de ce modèle hydropédologique est couplé avec un modèle déjà existant de la circulation générale atmosphé. rique. Les auteurs ont présenté les résultats d'une expérience de simulation d'une semaine d'Août qui paraissent montrer, pour trois régions sélectionnées des Etats-Unis, une concordance convenable avec les observations.

\section{Conclusion}

Depuis l'aventure de Noé, les hommes se sont beaucoup préoccupés de la relation ternaire pluie-infiltrationdébit. 
$\mathrm{Au}$ cours des dernières décennies, des investissements très importants en matériel et en temps ont été consacrés à ce problème. Mais il semble que nous ne disposions encore que de réponses fragmentaires ou trop rustiques. Ceci est dû, à notre avis, à la difficulté du changement d'échelle. A la dimension de l'échantillon homogène de laboratoire, les chercheurs maitrisent l'hydrodynamique du phénomène à condition de tenir compte de l'effet de l'air.. Mais, à notre connaissance, ils ne peuvent encore en déduire le comportement d'une parcelle ou d'un bassin versant expérimental mëme apparemment homogène. A l'échelle supérieure de la région, le géographe est presque obligé de se contenter de constatations statistiques.

Mais des essais intrépides comme ceux de l'équipe du Connecticut jettent déjà une passerelle entre hydrogéologie et météorologie et tentent une liaison encore balbutiante au niveau des trois échelles entre hydrodynamique, gestion des stocks et climats.

\section{Bibliographie restreinte}

FEODOROFF A. - Etude expérimentale de l'infiltration de l'eau non saturante. Thèse de Docteur-Ingénieur, INRA, Versailles, 87 pages, 1965 .

GREEN AMPT. - Studies on soil physics : I. Flow of air and water through soils. J. Agr. Sci., 4, 1-24, 1911.

LIN J.D., ALFANO JJ., BOCK P. - A large scale ground hydrologic model with interacts with the atmosphere. XVIII Congrès AIRH, Cagliari, Communication B.d.6, Septembre 1979.

MOREL-SEYTOUX H.J. - Pour une théorie réformée de l'infiltration. Pourquoi ? Cahier Hydrologique ORSTOM, Série Hydrologie X(2), pp. 185-194 X(3), pp. 199-209, 1973.

PHILIP J.R. - The theory of infiltration. Soil Science, 83, pp. $345-357 ; 84$, pp. $163-178$, pp. $257-264$, pp. $329-339$ 85, pp. $278-286,1957$.

THIRRIOT C., RIZK W. - Sur la pénétration de l'eau de précipitations successives dans un sol argileux. Réunion du groupe français des argiles 19/11/1970.

VACHAUD G. - Contribution à l'étude des problèmes d'écoulement en milieu poreux non saturé. Thèse ès Sciences, Université de Grenoble, 1968

\section{Discussion}

M. Le Président. - Merci pour cette remarquable mise au point. Y-a-t-il des questions ou des observations?

Personnellement je souhaiterais, à des fins pédagogiques, que toutes les planches et transparents présentés ici soient d'une manière ou d'une autre rendus publics, car ils constituent un excellent ensemble de documents pas toujours faciles à rassembler.
M. RODIER. - Il faut toujours se méfier des synthèses à l'échelle continentale.

M. THIRRIOT. - Je suis d'accord avec vous. Néanmoins le rapport présenté à l'AIRH à Kajari en Septembre dernier n'était pas inintéressant. 